Geociências

\title{
Suscetibilidade magnética na caracterização da variabilidade espacial de atributos do solo em solos subtropicais
}

\author{
Magnetic susceptibility in the characterization of spatial variability of \\ soils attributes in subtropical soils \\ Priscila Vogelei Ramos'®i, Ricardo Simão Diniz Dalmolin"(1), \\ Jean Michel Moura-Bueno"I(1), José Marques Junior"II(1),

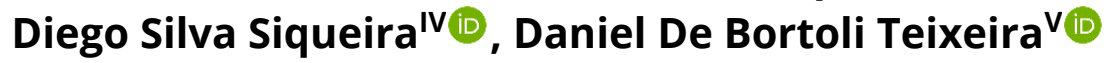 \\ ' Universidade de Burgos, Burgos, Espanha \\ "Universidade Federal de Santa Maria, Santa Maria, RS, Brasil \\ III Faculdade de Ciências Agrárias e Veterinárias, Jaboticabal, SP, Brasil \\ IV Universidade Estadual Paulista, Jaboticabal, SP, Brasil \\ $\vee$ Universidade de Marília, Marília, SP, Brasil
}

\section{RESUMO}

A Susceptibilidade magnética (SM) tem sido utilizada na estimativa de atributos do solo. Com a proposta de aumentar as informações de solos no sul do Brasil os objetivos deste trabalho foram: (i) avaliar a correlação da SM com atributos do solo em uma vertente de solos basálticos subtropicais; (ii) caracterizar a estrutura da variação espacial da SM e do teor de areia, argila e COS; e (iii) identificar a densidade amostral que capture a variabilidade espacial para auxiliar trabalhos futuros em condições semelhantes. Em uma área de 22 ha, no município de Santo Augusto - RS, Brasil, foi coletada uma malha amostral com 87 pontos para determinação dos atributos do solo. Também foram coletadas amostras em cindo perfis ao longo da vertente. Os dados dos perfis foram analisados pela correlação de Pearson para verificar o grau de correlação da SM com os atributos do solo. Na malha amostral foram realizadas análises de dependência espacial para avaliar grau da dependência espacial dos atributos. A SM apresentou alta correlação com os atributos do solo argila, $\mathrm{Fe}_{s}, \mathrm{Fe}_{\mathrm{d}}$ e COS. A avaliação da variação espacial mostrou que os atributos apresentaram grau de dependência espacial variando de fraco, para o COS, à forte para a SM. O padrão de variabilidade espacial sugere densidade amostral de um ponto a cada 4 a 12 ha.

Palavras-chave: Geoestatística; Densidade amostral; Pedometria 


\section{ABSTRACT}

Magnetic susceptibility (MS) has been used to estimate soil attributes. With the proposal to increase the information of soils in southern Brazil the objectives of this work were: (i) evaluate the correlation of SM with soil attributes in a slope of subtropical basaltic soils; (ii) to characterize the spatial variation structure of MS and the content of sand, clay and COS; and (iii) identify the sample density that captures the spatial variability to assist future work under similar conditions. In a 22 ha area located in Santo Augusto - RS, Brazil, an 87 points sample grid was collected to determine soil attributes. Samples were also collected in five profiles along the slope. The profile data were analyzed by correlation to verify the degree of Pearson correlation of the SM with the attributes of the soil. In the sample grid spatial dependence analyzes were performed to assess the degree of spatial dependence on soil attributes. The MS presented a high correlation with the attributes of clay soil, $\mathrm{Fe}_{s}, \mathrm{Fe}_{d}$ and $\mathrm{COS}$. The evaluation of the spatial variation structure showed that the attributes presented a degree of spatial dependence ranging from weak for COS to strong for MS. The spatial variability pattern suggests a sample density of one point every 4 to 12 ha.

Keywords: Geostatistics; Sample density; Pedometrics

\section{INTRODUÇÃO}

A demanda por informações espaciais de atributos do solo em diferentes escalas geográficas, especialmente no Brasil (DALMOLIN e TEN CATEN, 2015) tem aumentado devido a necessidade de desenvolver indicadores de qualidade do solo e impactos globais relacionados ao uso e ocupação do solo (FAO e ITPS, 2015; BÜNEMANN et al., 2018). Inventários detalhados da distribuição espacial de atributos do solo como carbono orgânico do solo (COS) (ZHANG et al., 2018) e textura do solo (CIAMPALINI et al., 2015) são essenciais para balizar questões como segurança alimentar, mudança climática e políticas ambientais (BÜNEMANN et al., 2018; LAL et al., 2018; SORENSEN et al., 2018).

Diante deste cenário Grunwald et al. (2015) destacaram que as pesquisas em ciência do solo devem focar no desenvolvimento de metodologias que permitam a predição de atributos do solo com qualidade de forma rápida e com menor custo. Nesse sentido, Camargo et al. (2018) observaram que técnicas que não utilizam reagentes químicos para obtenção de dados, associado a leitura das amostras com maior facilidade e rapidez, são abordagens sustentáveis que estão ganhando cada vez mais espaço. A análise da suscetibilidade magnética (SM) tem mostrado resultados promissores na identificação e descrição de ambientes pedogenéticos 
de diferentes locais como, na China (PINGGUO et al., 2016; GAO et al., 2019), no Oriente Médio (AYOUBI et al., 2018), Europa (BAUTISTA et al., 2018; HIRSCH et al., 2019) e nas diferentes regiões do Brasil: Nordeste (SANTOS et al., 2013), Norte (OLIVEIRA et al., 2015), Sudeste (MATIAS et al., 2015; SIQUEIRA et al., 2015; BARBOSA et al., 2019) e Sul (SILVA et al., 2010; RAMOS et al., 2017). Esses estudos têm abordado o uso da SM como ferramenta auxiliar na caracterização quantitativa de atributos do solo e da variabilidade espacial das mesmas.

A SM pode ser utilizada no desenvolvimento de assinaturas geomagnéticas para estudos detalhados sobre o solo (MATIAS et al., 2014) e na identificação de compartimentos representativos de ambientes pedogenéticos (RAMOS et al., 2017) por ser uma técnica sensível às variações mineralógicas do solo. Os principais minerais com expressão magnética estão na fração argila (maghemita e ferridrita ferrimagnética) (TORRENT et al., 2010), silte e areia fina (magnetita) (DEARING, 1999). Dessa maneira, os minerais com expressão magnética presentes nestas frações, podem ser detectados por meio dos valores da SM (SIQUEIRA et al., 2010, 2015). Além disso, os valores da SM podem ser considerados indicadores da pedodiversidade nos solos tropicais, onde os óxidos de ferro são os principais causadores da variabilidade físico-química dos solos. Assim, pode-se inferir que o mapa da SM pode expressar os processos pedogenéticos do solo na zona de clima tropical (CAMARGO et al., 2014; SIQUEIRA et al., 2015).

A SM também é capaz de estimar indiretamente a o teor de COS. Na região tropical, ocorre redução de $80 \%$ da matéria orgânica do solo após cinco anos de cultivo agrícola convencional em solos menos de 15\% de teor de argila, 76\% para solos com teor de argila entre $15-30 \%$ e, $46 \%$ para solo com mais de $30 \%$ de teor de argila (SILVA et al, 1994). A elevada estabilidade da matéria orgânica em solos com maior teor de argila vem sendo atribuída aos maiores teores de minerais presentes nessa fração do solo como os óxidos de ferro hematita e goethita, e hidróxidos de alumínio, como a gibbsita, que complexam a matéria orgânica, estabilizando-a (ROSCOE et al., 2000; DALMOLIN et al., 2006; KLEBER et al., 2007). 
Sabendo-se que a SM é influenciada pela composição dos minerais do solo, leituras da SM podem expressar ambientes com diferentes potencias de oxidação e redução da matéria orgânica, consequentemente, fornecer estimativas dos teores de COS (OLIVEIRA et al., 2015). Outra maneira de associar indiretamente a SM com o COS é por meio de diferentes teores de matéria orgânica e emissão de $\mathrm{CO}_{2}$. Correlações entre a $\mathrm{SM}$ e emissão de $\mathrm{CO}_{2}$ foram encontras em Latossolos tropicais brasileiros com diferentes teores de matéria orgânica por Leal et al. (2015).

Diante da necessidade de gerar mapas detalhados do teor de areia, argila e COS por meio de técnicas rápidas, precisas e de menor custo, existe a necessidade de estudos que avaliem a magnitude da correlação da SM com a granulometria e $\cos$ e, se essa correlação associada a estrutura da variação espacial permite utilizar a SM para estimar indiretamente esses atributos em solos de origem basáltica no Sul do Brasil. Nesse sentido, os objetivos deste trabalho foram: (i) avaliar a correlação da SM com atributos do solo em uma vertente de solos basálticos subtropicais; (ii) caracterizar a estrutura da variação espacial da SM e do teor de areia, argila e COS; e (iii) identificar a densidade amostral que capture a variabilidade espacial para auxiliar trabalhos futuros em condições semelhantes.

\section{METODOLOGIA}

O estudo foi realizado em uma área de 22 ha, no município de Santo Augusto localizado ao norte do Estado do Rio Grande do Sul, Brasil (Figura 1a e 1b). O clima da região segundo a classificação de Köppen é do tipo Cfa - subtropical (ALVARES et al., 2013). O relevo predominantemente é suave ondulado, com declividade variando entre 3 a $8 \%$. As altitudes variam entre 455 a 495 metros (Figura 1b). $\mathrm{Na}$ região há predomínio de rochas vulcânicas extrusivas básicas (basalto) derivadas da Formação Serra Geral (WILDNER et al., 2008). 
Figura 1 - Localização do município de Santo Augusto no Estado do Rio Grande do Sul (a), área experimental detalhando a malha de amostragem (b) e perfil de elevação com a posição dos perfis dos solos descritos (c)

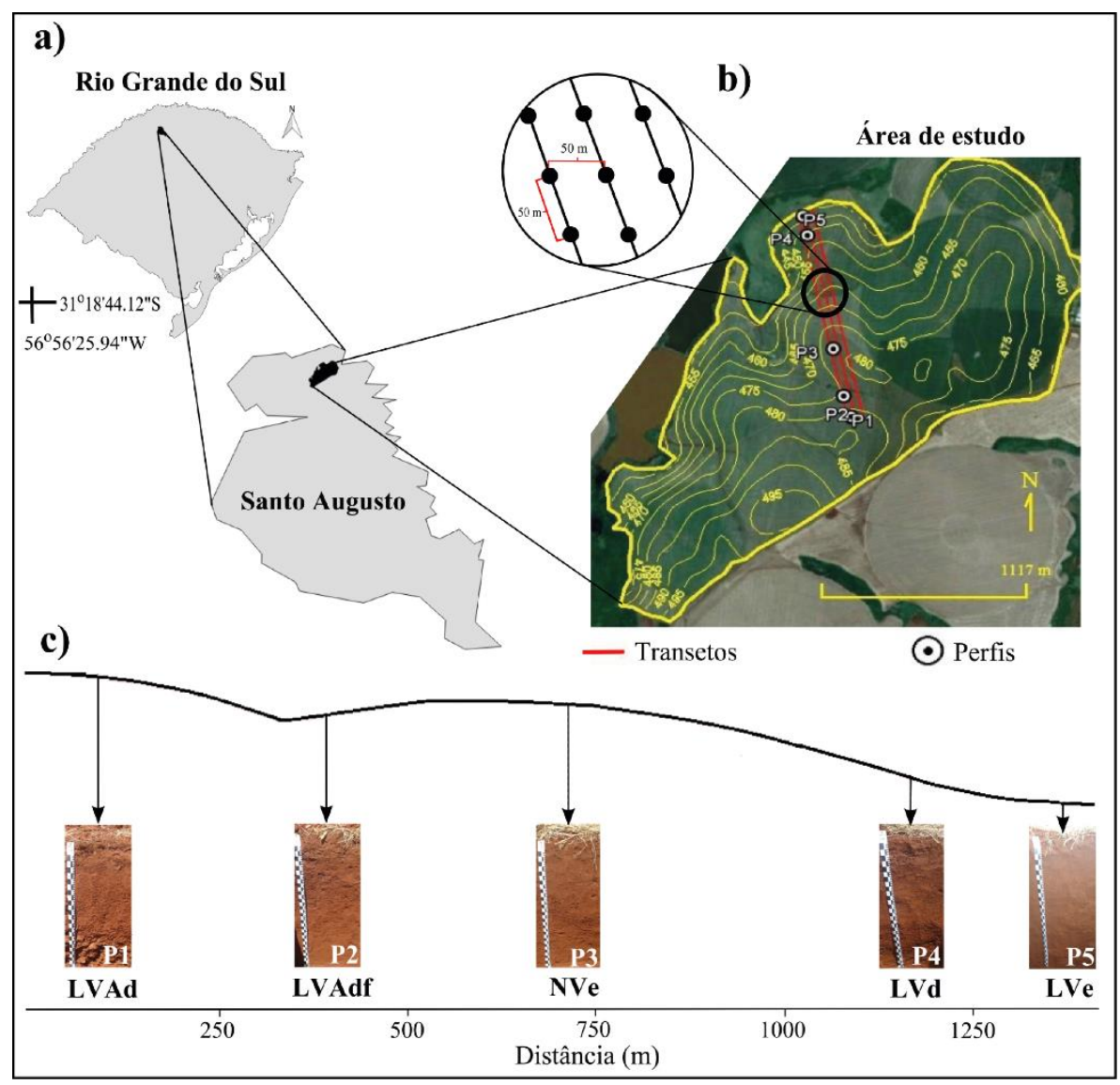

Para caracterização pedológica da área e análise de correlação da SM e atributos do solo, foram descritos e amostrados cinco perfis de solos conforme Santos et al. (2015), em locais representativos dos compartimentos da vertente (Figura 1b e 1c). Os solos foram classificados como LATOSSOLO VERMELHOAMARELO Distrófico típico - LVAd (P1), LATOSSOLO VERMELHO-AMARELO Distroférrico típico -LVAdf (P2), NITOSSOLO VERMELHO Eutrófico latossólico NVe (P3), LATOSSOLO VERMELHO Distrófico típico -LVd (P4) e LATOSSOLO VERMELHO Eutrófico típico - LVe (P5) de acordo com o Sistema Brasileiro de Classificação de Solos (SANTOS et al., 2018). Nas amostras dos perfis foram determinados os teores de areia, silte, argila, $\mathrm{COS}$, alumínio trocável $\left(\mathrm{Al}^{3+}\right)$, acidez potencial $\left(\mathrm{H}+\mathrm{Al}{ }^{+3}\right), \mathrm{pH}$ em água e em $\mathrm{KCl}$, teores de potássio $(\mathrm{K})$, cálcio $(\mathrm{Ca})$, magnésio $(\mathrm{Mg})$ e ferro extraído 
com ácido sulfúrico $\left(\mathrm{Fe}_{\mathrm{s}}\right.$ ) segundo metodologia de Teixeira et al. (2017). Os teores de ferro cristalino $\left(\mathrm{Fe}_{\mathrm{d}}\right)$ e ferro de baixa cristalinidade $\left(\mathrm{Fe}_{\mathrm{o}}\right)$ foram determinados segundo metodologia preconizada por Mehra e Jackson (1960) e McKeague e Day (1966), respectivamente. A SM foi determinada em 10 gramas da fração argila, areia fina e TFSA dos perfis, utilizando o equipamento MS2 (Bartington Instruments Ltd., Oxford, Inglaterra), acoplado a um sensor de laboratório MS2B de duas frequências, conforme metodologia descrita por Dearing (1999).

Para o estudo da variabilidade espacial dos atributos do solo areia, argila, COS e SM, foram demarcados três transetos de 1.450 m cada um. Em cada transeto foram coletados 29 pontos, totalizando 87 pontos com distância de 50 metros entre si, resultando em uma densidade amostral de cinco pontos ha-1 (Figura 1b). O planejamento amostral segue proposta de estudos anteriores para caracterização da variabilidade espacial de atributos do solo, os quais observaram máxima variação ao longo da vertente (CAMARGO et al., 2015; SIQUEIRA et al., 2015). Em cada ponto da malha foram coletadas amostras nas profundidades de 0,000,05 m, 0,05-0,15 m, 0,15-0,30 m e 0,30-0,60 m, conforme proposição do GlobalSoilMap (ARROUAYS et al., 2014), totalizando 348 amostras. Após secas ao ar, moídas e peneiradas em malha de $2 \mathrm{~mm}$ (TFSA) foram determinados nestas amostras a granulometria e o teor de COS, conforme metodologia descrita em Teixeira et al. (2017). A SM foi determinada utilizando 10 gramas de TFSA de cada um dos pontos amostrais por um medidor de suscetibilidade MS2.

Nas amostras coletadas dos perfis, foi realizada a análise de correlação linear de Pearson nos horizontes superficiais (Ap - profundidade entre 0,00-0,30 m) e subsuperficiais (Bw1-profundidade entre 0,60-1,36 m) para verificar o grau de correlação da SM com os atributos do solo (areia, argila, COS, $\mathrm{Fe}_{s}, \mathrm{Fe}_{d}$ e Feo). Nas amostras da malha amostral para os atributos do solo, areia, argila, COS e SM foram realizadas análises de comparações de média (Teste de Tukey) e dependência espacial para cada profundidade do solo. Para verificar a dependência espacial dos 87 pontos coletados, foi utilizada a análise geoestatística 
com base na modelagem e ajustes dos semivariogramas. O semivariograma experimental foi determinado por meio do cálculo da variância em razão da distância de separação entre amostras (Equação 1):

$$
\hat{\gamma}(h)=\frac{1}{2 \mathrm{~N}(h)} \sum_{\alpha=1}^{\mathrm{N}(h)}\left[z\left(x_{i}+h\right)-z\left(x_{i}\right)\right]^{2}
$$

em que $\hat{\gamma}(h)$ é a variância experimental para uma distância de separação $h$, $N(h)$ é o número de pares experimentais de observações $Z\left(x_{i}\right)$ e $Z\left(x_{i}+h\right)$ são separados pela distância h.

Foram testados os modelos esféricos, exponenciais e gaussianos. Tais modelos foram ajustados aos semivariogramas experimentais, possibilitando a definição dos parâmetros: efeito pepita $\left(C_{0}\right)$, patamar $\left(C_{0}+C_{1}\right)$, alcance e cálculo do grau de dependência espacial. De posse do alcance de cada atributo do solo é possível estabelecer até quantos metros, apenas uma amostra terá influência, podendo inferir a densidade amostral de determinado atributo em determinadas condições. Para o cálculo da densidade amostral basta elevar ao quadrado o alcance $(\mathrm{m})$ e em seguida dividir por $10.000 \mathrm{~m}^{2}$ (1 ha). Para analisar o grau da dependência espacial (GDE) dos atributos utilizou-se a classificação de Cambardella et al. (1994), em que são considerados de dependência espacial forte os semivariogramas que têm um efeito pepita $<25 \%$ do patamar, moderada quando está entre 25 e $75 \%$ e fraca, > $75 \%$.

O ajuste e modelagem dos variogramas foram baseados no coeficiente linear e angular obtidos nos procedimentos de validação cruzada. Na validação cruzada, cada valor observado é removido e, posteriormente, estimado, utilizando os demais valores (LAMDIM, 2006). Em todos os semivariogramas modelados, foram obtidos pares de pontos suficientes (acima de 50) para as estimativas das semivariâncias (GOOVAERTS, 1997). Os cálculos e a modelagem dos semivariogramas foram realizados por meio do software GS+ v.9.0 (GAMMA DESIGN DE SOFTWARE, 2008). 


\section{RESULTADOS E DISCUSSÃO}

A SM apresentou correlação positiva moderada com os teores de argila ( $r=$ $0,66$ e $r=0,63 ; p<0,05)$ e teores de $\cos (r=0,61$ e $r=0,60 ; p<0,05)$ nos horizontes superficiais e subsuperficiais (Tabela 1). Souza Junior et al. (2010) estudando horizontes B em diferentes solos, desenvolvidos predominantemente de rochas basálticas, no Estado do Paraná, verificaram correlação significativa entre a SM e argila $(r=0,98 ; p<0,01)$, indicando a provável presença de minerais superparamagnéticos. A correlação da SM com o COS mostra a estreita relação que o comportamento magnético tem com solos com elevada estabilidade da matéria orgânica. Esta estabilidade está atrelada a solos de textura argilosa e, consequentemente, com os minerais de maior expressão magnética na fração argila, como é o caso dos Latosssolos Vermelhos da área de estudo (RAMOS et al., 2017). Esta significativa correlação concorda com os resultados obtidos por Barbosa (2014) em Latossolos, que também verificou a existência de correlação positiva $(r=0,41 ; p<0,05)$ na camada superficial do solo $(0,00-0,25 \mathrm{~m})$.

Os teores da areia apresentaram correlação negativa forte com a SM no horizonte superficial $(r=-0,85 ; p<0,01)$ e subsuperficial $(r=-0,80 ; p<0,05)$ (Tabela 1). Tal resultado está relacionado ao menor conteúdo de minerais ferrimagnéticos na fração areia. A correlação entre a SM e os teores de ferro no solo $\mathrm{Fe}_{\mathrm{s}}(r=0,91$; $p<0,01)$ e $\mathrm{Fe}_{d}(r=0,77 ; p<0,05)$ apresentaram altas correlações e significativas. As maiores correlações observadas entre a $\mathrm{SM}$ e $\circ \mathrm{Fe}_{\mathrm{s}}$ e $\mathrm{Fe}_{d}$ estão relacionadas a presença de minerais litogenéticos e pedogenéticos de expressão magnética como magnetita, maghemita e ferridrita ferrimagnética, proporcionando, deste modo, uma correlação positiva forte (RAMOS et al., 2017). Já a correlação menor e não significativa é observada para o $\mathrm{Fe}_{\mathrm{o}}(r=0,18)$ está associada a formas de ferro mal cristalizados (amorfos) que apresenta baixa atração ferromagnética. As correlações positivas dos teores de ferro com a SM concordam com resultados 
encontrados por Siqueira et al. (2010) em Argissolos originários de sedimentos areníticos, e Matias et al. (2014) em Latossolos originados de arenito e basalto.

Tabela 1 - Correlação linear de Pearson entre os atributos físicos, químicos e a suscetibilidade magnética (SM) nos horizontes superficial e subsuperficial dos cinco perfis de solo

\begin{tabular}{lcc}
\hline $\begin{array}{l}\text { Atributos } \\
\left(\mathbf{g ~ k g}^{-1}\right)\end{array}$ & $\begin{array}{c}\text { Horizonte Ap } \\
\text { (profundidade entre 0,00-0,30 m) }\end{array}$ & $\begin{array}{c}\text { Horizonte Bw1 } \\
\text { (profundidade entre 0,60-1,36 m) }\end{array}$ \\
\hline Areia & $-0,85^{* *}$ & $-0,80^{*}$ \\
Argila & $0,66^{*}$ & $0,63^{*}$ \\
$\mathrm{Fe}_{\mathrm{s}}$ & $0,91^{* *}$ & $0,84^{* *}$ \\
$\mathrm{Fe}_{\mathrm{d}}$ & $0,77^{*}$ & $0,61^{*}$ \\
$\mathrm{Fe}_{\mathrm{o}}$ & $0,18^{\mathrm{ns}}$ & $0,32^{\mathrm{ns}}$ \\
$\mathrm{COS}$ & $0,61^{*}$ & $0,60^{*}$ \\
\hline
\end{tabular}

$\mathrm{Fe}_{\mathrm{s}}$ - ferro extraído com ácido sulfúrico; $\mathrm{Fe}_{\mathrm{d}}$ - ferro extraído com ditionito, citrato e bicarbonato sódio; $\mathrm{Fe}_{\text {。 }}$ - ferro extraído com oxalato ácido de amônio; COS - carbono orgânico do solo; * - Significativo a 5\% de probabilidade; ** - Significativo a $1 \%$ de probabilidade; ns - Não Significativo.

Todos os atributos apresentaram diferença significativa em profundidade na comparação das médias para as 348 amostras (Tabela 2). Os teores dos atributos na camada superficial (0,00-0,05 e 0,05-0,15 m) provavelmente foram influenciados pelo sistema de manejo e processo erosivo, que são mais atuantes nessas camadas, principalmente para o COS, enquanto que, na camada mais profunda $(0,15-0,30$ e 0,30-0,60 m) os atributos se mantiveram mais estáveis, não diferindo pelo teste de Tukey. O teor de COS foi mais elevado em superfície devido ao maior aporte de resíduos culturais nessa camada. Os valores de argila aumentaram em profundidade e de areia reduziram, seguindo a distribuição granulométrica típica de Latossolos e Nitossolos do Planalto do Rio Grande do Sul (STRECK et al., 2018). 
Tabela 2 - Estatística descritiva dos atributos dos solos na malha de amostragem de 87 pontos, nas quatro profundidades

\begin{tabular}{|c|c|c|c|c|c|c|c|}
\hline Atributo & Profundidade (m) & Média & Min. & Max. & DP & CV & Assimetria \\
\hline \multirow{4}{*}{$\begin{array}{l}\mathrm{SM} \\
10^{-6} \mathrm{~m}^{3} \mathrm{~kg}^{-1}\end{array}$} & $0,00-0,05$ & $37,3 \mathrm{~b}$ & 18,2 & 47,0 & 7,6 & 20,3 & $-0,8$ \\
\hline & $0,05-0,15$ & 37,5 b & 18,3 & 48,1 & 7,9 & 20,6 & $-1,0$ \\
\hline & $0,15-0,30$ & $38,7 \mathrm{a}$ & 16,3 & 50,0 & 8,4 & 21,6 & $-1,1$ \\
\hline & $0,30-0,60$ & $38,4 \mathrm{a}$ & 17,3 & 47,9 & 8,1 & 21,0 & $-1,0$ \\
\hline \multirow{4}{*}{$\begin{array}{l}\text { COS } \\
\mathrm{g} \mathrm{kg}^{-1}\end{array}$} & $0,00-0,05$ & $22,8 \mathrm{a}$ & 11,0 & 34,0 & 4,4 & 19,3 & 0,2 \\
\hline & $0,05-0,15$ & $16,4 \mathrm{~b}$ & 7,0 & 25,0 & 2,8 & 17,1 & $-0,1$ \\
\hline & $0,15-0,30$ & $14,1 \mathrm{c}$ & 7,0 & 17,0 & 2,3 & 16,3 & $-0,9$ \\
\hline & $0,30-0,60$ & $12,9 c$ & 8,0 & 18,0 & 3,3 & 25,6 & 2,0 \\
\hline \multirow{4}{*}{$\begin{array}{l}\text { Argila } \\
\text { g kg-1 }^{-1}\end{array}$} & $0,00-0,05$ & $599,6 \mathrm{c}$ & 420,0 & 710,3 & 68,6 & 11,4 & $-0,9$ \\
\hline & $0,05-0,15$ & $653,5 \mathrm{~b}$ & 473,5 & 770,2 & 53,3 & 8,2 & $-1,0$ \\
\hline & $0,15-0,30$ & $669,1 \mathrm{ab}$ & 510,0 & 801,0 & 53,2 & 7,9 & $-0,9$ \\
\hline & $0,30-0,60$ & $679,5 \mathrm{a}$ & 371,0 & 772,0 & 76,9 & 11,3 & $-1,9$ \\
\hline \multirow{4}{*}{$\begin{array}{l}\text { Areia } \\
\mathrm{g} \mathrm{kg}^{-1}\end{array}$} & $0,00-0,05$ & 99,7 a & 40,0 & 230,3 & 36,0 & 36,1 & 1,3 \\
\hline & $0,05-0,15$ & 81,7 b & 40,0 & 210,3 & 31,8 & 38,9 & 1,4 \\
\hline & $0,15-0,30$ & $71,0 \mathrm{c}$ & 30,0 & 210,1 & 29,9 & 42,1 & 1,6 \\
\hline & $0,30-0,60$ & $67,2 c$ & 30,0 & 210,0 & 30,9 & 45,9 & 1,9 \\
\hline
\end{tabular}

Min. - valor mínimo; Máx. - valor máximo; DP - desvio padrão; CV - coeficiente de variação; COS - carbono orgânico do solo; SM - suscetibilidade magnética. Médias seguidas pela mesma letra não diferem entre si pelo teste de Tukey, ao nível de $5 \%$ de probabilidade.

Os valores de SM variaram de 16 a 50,0 10-6 $\mathrm{m}^{3} \mathrm{~kg}^{-1}$, valores esses próximos aos encontrados por Barbosa et al. (2019) em solos derivados de basalto no nordeste do Estado de São Paulo $\left(5,1\right.$ a 74,6 $\left.10^{-6} \mathrm{~m}^{3} \mathrm{~kg}^{-1}\right)$. Os resultados de SM também se enquadram a faixa de assinatura magnética encontrada por Silva et al. (2010) na região sudoeste do Paraná (60 a $1510^{-6} \mathrm{~m}^{3} \mathrm{~kg}^{-1}$ ). Essa similaridade com as leituras de Silva et al. (2010) é influência da continuidade geológica entre os Estados do RS e PR. Os valores mais elevados de SM foram identificados em 
maiores profundidades, este mesmo comportamento foi observado na fração argila. Esse comportamento provavelmente ocorre devido a presença de minerais de expressão magnética nessa fração, como maghemita e ferridrita ferrimagnética (MICHEL et al., 2010; TORRENT et al., 2010; POGGERE et al., 2018). Para os solos da região de estudo, a máxima expressão dos processos pedogenéticos, ocorrem no horizonte B (KÄMPF e CURI, 2012), consequentemente, ocorre o maior teor desses minerais ferromagnéticos na fração argila, com destaque para os Latossolos e Nitossolos (DALMOLIN et al., 2006). Isso é confirmado em estudo de Ramos et al. (2017), que mostrou maior concentração desses minerais na fração argila do horizonte B de Latossolos e Nitossolos região o Planalto do Rio Grande do Sul.

Na Figura 2 são apresentados os semivariogramas experimentais e modelos ajustados, dos 87 pontos nas quatro profundidades. Não foi observada estrutura de dependência espacial para a argila nas profundidades 0,05-0,15 e 0,30-0,60 m, apresentando assim, o efeito pepita puro. De acordo com Yamamoto e Landim (2013) esta situação pode ser atribuída a erros de medição ou a intervalos de coleta insuficientes para mostrar o comportamento espacial do fenômeno em estudo. No presente estudo, tal resultado está associado ao intervalo de amostragem, apesar da variação do teor de argila na área ser baixo, apresentou maior amplitude nessas duas profundidades (Tabela 2). Para captar a estrutura de dependência espacial neste caso, seria preciso uma amostragem mais densa na área, o que só pode ser obtido apenas em estudos mais detalhado, confirmando esta hipótese.

O modelo esférico apresentou melhor ajuste à estrutura da variabilidade espacial dos atributos avaliados (Figura 2), com destaque para a SM, corroborando com estudo de Oliveira et al. (2015). Esse modelo indica que a estrutura da variação espacial apresenta comportamento abrupto, ou seja, existe uma transição abrupta dos valores modelados ao longo da paisagem, as quais geralmente estão associadas aos fatores de formação do solo como transição geológica e ou compartimentos da paisagem (SIQUEIRA et al., 2015). Neste estudo essa transição está relacionada ao segundo fator conforme observado por Ramos et al. (2017) em 
Latossolos subtropicais e Matias et al. (2015) e Latossolos tropicais. A adequação desse modelo está de acordo com outros estudos da literatura, sendo o mais utilizado para a descrição da variabilidade espacial dos atributos do solo (BITENCOURT et al., 2016; GHORBANZADEH et al., 2019).

A avaliação da estrutura da variação espacial mostrou que os atributos apresentaram grau de dependência espacial variando de fraco (80\%), para o COS na profundidade de $0,00-0,05 \mathrm{~m}$, à forte (0\%) para a SM em todas as profundidades avaliadas, segundo classificação proposta por Cambardella et al. (1994). A fraca dependência espacial observada para o COS está relacionada a maior variação inerente desse atributo do solo causada por práticas de manejo, com destaque para a profundidade de 0,00-0,05 m (Tabela 2). Santos et al. (2013) obtiveram resultados semelhantes em Neossolos e Argissolos do Estado do Piauí, onde a SM apresentou dependência espacial forte, com valores próximos de $0 \%$.

Variáveis que evidenciam forte dependência espacial são mais influenciadas por propriedades intrínsecas do solo, ou seja, pelos fatores pedogenéticos (CAMBARDELLA et al., 1994). Já dependência espacial moderada ou fraca, possivelmente seria em razão da homogeneização do solo causada por prática de manejo. Isso explica porque a SM apresentou grau de dependência espacial forte e o COS grau fraco no presente estudo. Além disso, a área de estudo apresenta relevo com formato côncavo, o qual favorece a erosão e transporte e material, que influenciam na variabilidade espacial de atributos do solo (SOUZA et al., 2003). A região do Planalto de estado do Rio Grande do Sul é caracterizada por pedoformas convexas que condicionam maior remoção nas partes mais altas e maior acúmulo nas áreas mais baixas. Portanto, essas diferentes posições criam situações locais diferenciadas na estrutura da variação espacial, resultado na necessidade de diferentes designers amostrais para capturar tal variação conforme o atributo do solo investigado. 
Figura 2 - Semivariogramas da suscetibilidade magnética (SM), teores de carbono orgânico do solo (COS), argila e areia nas quatro profundidades avaliadas. Esf. ( $\mathrm{C}_{0} ; \mathrm{C}_{0}+$ $\left.\mathrm{C}_{1} ; \mathrm{GDE}(\%) ; \mathrm{A}(\mathrm{m}) ; \mathrm{a} ; \mathrm{b}\right), \mathrm{Esf}=$ modelo esférico; $\mathrm{C}_{0}=$ efeito pepita; $\mathrm{C}_{0}+\mathrm{C}_{1}=$ patamar; GDE = grau de dependência espacial; $\mathrm{A}=$ alcance; $\mathrm{a}=$ coeficiente linear; $\mathrm{b}=$ coeficiente angular; EPP = efeito pepita puro. $\mathrm{O}$ tracejado representa a variância das amostras e as linhas contínuas o modelo de semivariograma ajustado

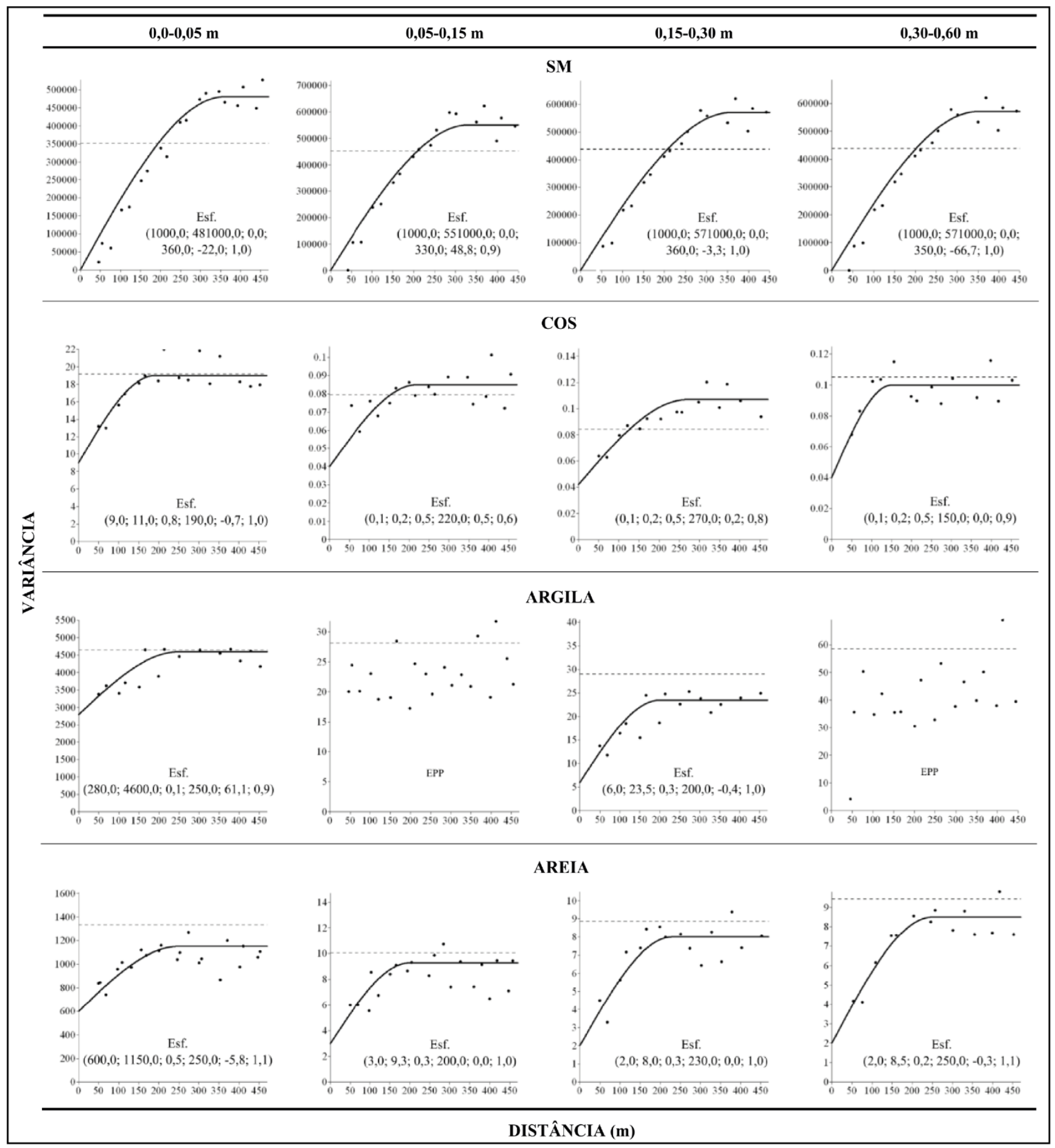


O alcance médio dos atributos estudados são $243 \mathrm{~m}$ para areia, $225 \mathrm{~m}$ para argila, 207 m para o COS e 350 m para a SM. Este padrão de variabilidade sugere densidade amostral de um ponto a cada 6 ha suficiente para caracterizar a variabilidade espacial da areia, um ponto a cada 5 ha para a argila, um ponto a cada 4 ha para o COS e um ponto a cada 12 ha para a SM. Esse resultado mostra que a SM é o atributo que necessita de um menor número de amostras, visto que a densidade amostral pode ser menor e mesmo assim contemplar a escala de variação espacial inerente ao atributo. No entanto, neste estudo os alcances foram calculados para uma malha de amostragem ao longo de uma vertente. Isso deve ser levado em consideração, pois esse fator tem influência na anisotropia conforme observado por Souza et al. (2003). Os autores encontraram alcance duas vezes maior no sentido do declive em relação ao sentido das maiores cotas de altimetria para atributos químicos e físicos de Latossolo basáltico em uma vertente na região Centro-Oeste do Estado de São Paulo. Oliveira et al. (2015) também encontram maiores valores de alcance para a SM em relação aos atributos argila, areia e COS para Argissolos da Amazônia nas profundidades de 0,0-0,20 e 0,40-0,60 $\mathrm{m}$.

Por fim, o uso SM mostrou ter potencial para ser utilizada como variável auxiliar no mapeamento detalhado dos atributos do solo como, areia, argila e COS e no planejamento de designers de amostragem. Isso pode refletir na redução do tempo e custo empregado nas etapas de amostragem e análises físicas e químicas. Ainda, essa abordagem pode ser empregada para obtenção de mapas digitais de solos de alta resolução, sendo esses úteis para um melhor aproveitamento dos recursos naturais, considerando os diferentes sistemas de produção e sua codependência com os solos. 


\section{CONCLUSÕES}

A SM apresentou correlação significativa com os atributos do solo argila, $\mathrm{Fe}_{\mathrm{s}}$, $\mathrm{Fe}_{d}$ e COS, indicando que a SM pode ser utilizada para estimá-los indiretamente.

A estrutura de variação espacial encontrada entre a SM e atributos do solo mostra que a SM tem potencial para aplicação no mapeamento detalhado desses atributos em solos subtropicais do Planalto do Rio Grande do Sul.

Com base na variação espacial encontrada, sugere-se a densidade amostral de um ponto a cada 4 a 12 ha para capturar a variabilidade espacial de atributos de solos subtropicais em condições ambientais semelhantes.

\section{REFERÊNCIAS}

ALVARES, C. A.; STAPE, J. L.; SENTELHAS, P. C.; GONÇALVES, J. L. M.; SPAROVEK, G. Köppen's climate classification map for Brazil. Meteorologische Zeitschrift, v. 22, n. 6, p. 711-728, 2013.

ARROUAYS, D.; MCKENZIE, N.; HEMPEL, J.; FORGES, A. R.; McBRATNEY, A. GlobalSoilMap: Basis of the Global Spatial Soil Information System. CRC Press/Balkema; 2014.

AYOUBI, S.; ABAZARI, P.; ZERAATPISHEH, M. Soil great groups discrimination using magnetic susceptibility technique in a semi-arid region, central Iran. Arab J Geosci, v. 11, p. 616, 2018.

BARBOSA, R. S. Erodibilidade de Latossolos predita pela suscetibilidade magnética e espectroscopia de reflectância difusa 2014. 78 p. Tese (Doutorado em Ciência do Solo) Faculdade de Ciências Agrárias e Veterinárias/Universidade Estadual Paulista "Julio de Mesquita Filho", Jaboticabal, São Paulo, 2014.

BARBOSA, R.S.; MARQUES JÚNIOR, J.; BARRÓN, V.; MARTINS FILHO, M. V.; SIQUEIRA, D. S.; PELUCO, R. G.; CAMARGO, L. A.; SILVA, L. S. Prediction and mapping of erodibility factors (USLE and WEPP) by magnetic susceptibility in basalt-derived soils in northeastern São Paulo state, Brazil. Environmental Earth Sciences, v. 78, p. 12, 2019.

BAUTISTA, F.; BÓGALO, M. F.; NAVARRO, A. S.; GOGUITCHAICHVILI, A.; INIESTA, M. J. D.; CEJUDO, R.; SANLEANDRO, P. M.; GIL, J. M.; DÍAZ-PEREIRA, E. Magnetic and pedological characterisation of a paleosol under aridic conditions in Spain. Studia Geophysica et Geodaetica, v. 62, p. 139166, 2018. 
BITENCOURT, D. G. B.; BARROS, W. S.; TIMM, L. C.; SHE, D.; PENNING, L. H.; PARFITT, J. M. B.; REICHARDT, K. Multivariate and geostatistical analyses to evaluate lowland soil levelling effects on physico-chemical properties. Soil and Tillage Research, v. 156, p. 63-73, 2016.

BÜNEMANN, E. K.; BONGIORNO, G.; ZHANGUO, B.; CREAMER, R. E.; DEYN, G. D.; GOEDE, R.; FLESKENS, L.; GEISSEN, V.; KUYPER, T. W.; MÄDER, P.; PULLEMAN, M.; SUKKEL, W.; GROENIGEN, W. VAN.; BRUSSAARD, L. Soil quality - A critical review. Soil Biology and Biochemistry, v. 120, p. 105-125, 2018.

CAMARGO, L. A.; MARQUES JUNIOR, J.; PEREIRA, G. T.; BAHIA, A. S. R. S. Clay mineralogy and magnetic susceptibility of Oxisols in geomorphic surfaces. Scientia Agricola, v. 71, p. 244-256, 2014.

CAMARGO, L. A.; MARQUES JUNIOR, J.; BARRÓN, V.; ALLEONI, L. R. F.; BARBOSA, R. S.; PEREIRA, G. T. Mapping of clay, iron oxide and adsorbed phosphate in Oxisols using diffuse reflectance spectroscopy. Geoderma, v. 251, p. 124-132, 2015.

CAMARGO, L. A.; MARQUES JUNIOR, J.; BARRÓN, V.; ALLEONI, L. R. F.; PEREIRA, G. T.; TEIXEIRA, D. B. et al. Predicting potentially toxic elements in tropical soils from iron oxides, magnetic susceptibility and diffuse reflectance spectra. Catena, v. 165, p. 503-515, 2018.

CAMBARDELLA, C. A.; MOORMAN, T. B.; NOVAK.; J. M.; PARKIN, T. B.; KARLEN, D. L.; TURCO, R. F.; KONOPKA, A. E. Field-scale variability of soil properties in central lowa soils. Soil Science Society of America Journal, v. 58, p. 1501-1511, 1994.

CIAMPALINI, A.; ANDRÉ, F.; GARFAGNOLI, F.; GRANDJEAN, G.; LAMBOT, S.; CHIARANTINI, L.; MORETTI, S. Improved estimation of soil clay content by the fusion of remote hyperspectral and proximal geophysical sensing. Journal of Applied Geophysics, v. 116, p. 135-145, 2015.

DALMOLIN, R. S. D.; GONÇALVES, C. N.; DICK, D. P.; KNICKER, H.; KLAMT, E.; GEL-KNABNER, I. Organic matter characteristics and distribution in ferralsol profiles of a climosequence in southern Brazil. European Journal Soil Science, v. 57, n. 4, p. 644-654, 2006.

DALMOLIN, R. S. D.; TEN CATEN, A. Mapeamento Digital: nova abordagem em levantamento de solos. Investigación Agraria, v. 17, n. 2, p. 77-86, 2015.

DEARING, J A. Environmental magnetic susceptibility: using the Bartington MS2 system. 2 ed. Kenilworth: Chi Publishing; 1999.

FAO, ITPS - Food and Agriculture Organization of the United Nations, Intergovernmental Technical Panel on Soils. Status of the World's Soil Resources (SWSR) - Main Report. Rome, Italy; 2015. Disponível em: http://www.fao.org/3/i5199e/l5199E.pdf.

GAMMA DESIGN SOFTWARE - GS+: Geostatistics for the Enviromental Sciences, Version 9.0. Gamma Design Software. Michigan, USA; 2008. 
GAO, X.; HAO, Q.; OLDFIELD, F.; BLOEMENDAL, J.; DENG, C.; WANG, L.; SONG, Y.; GE, J.; WU, H.; $X U$, B.; LI, F.; HAN, L.; FU, Y.; GUO, Z. New high-temperature dependence of magnetic susceptibility-based climofunction for quantifying paleoprecipitation from Chinese loess. Geochemistry Geophysics Geosystems, v. 20, p. 4273-4291, 2019.

GHORBANZADEH, N.; SAlEHI, A.; POURBABAEI, H.; TOLAROD, A. A. S.; ALAVI, S. J. Spatial variability of soil microbial indices in common alder COMMON ALDER (Alnus glutinosa) stands using a geostatistical approach in northern Iran. Journal of Forestry Research, v. 30, p. 679-688, 2019.

GOOVAERTS, P. Geostatistics for Natural Resources Evaluation. Oxford University Press: NewYork; 1997.

GRUNWALD, S.; VASQUES, G. M.; RIVERO, R. G. Fusion of soil and remote sensing data to model soil properties. In: SPARKS DL, editors. Advances in Agronomy. Elsevier, vol. 131, p. 1-109, 2015.

HIRSCH, F.; BONHAGE, A.; BAURIEGEL, A.; SCHNEIDER, A.; RAAB, T.; RAAB, A.; GYPSER, S. The occurrence, soil parameters and genesis of rubified soils ('Fuchserden') of northeastern Germany. Catena, v. 175, p. 175:77-92, 2019.

KÄMPF, N.; CURI, N. Formação e evolução do solo (Pedogênese). In: KER, J.C.; CURI, N.; SCHAEFER, C.E.; VIDAL-TORRADO, P. (Eds.) Pedologia: Fundamentos. Sociedade Brasileira de Ciência Solo: Viçosa, MG, p. 207-302, 2012.

KLEBER, M.; SOLLINS, P.; SUTTON, R. A conceptual model of organo-mineral interactions in soils: self-assembly of organic molecular fragments into zonal structures on mineral surfaces. Biogeochemistry, v. 85, p. 9-24, 2007.

LAL, R.; SMITH, P.; JUNGKUNST, H. F.; MITSCH, W. J.; LEHMANN, J.; NAIR, P. K. R.; et al. The carbon sequestration potential of terrestrial ecosystems. Journal of Soil and Water Conservation, v. 73, n. 6, p. 145A-152A, 2018.

LEAL, F. T.; FRANÇA, A. B. C.; SIQUEIRA, D. S.; TEIXEIRA, D. B.; MARQUES JUNIOR, J.; LA SCALA, N. J. Characterization of potential $\mathrm{CO}_{2}$ emissions in agricultural areas using magnetic susceptibility. Scientia Agricola, v. 72, p. 535-539, 2015.

MATIAS, S. S. R.; MARQUES JUNIOR, J.; SIQUEIRA, D.S.; PEREIRA, G.T. Outlining precision boundaries among areas with different variability standards using magnetic susceptibility and geomorphic surfaces. Engenharia Agrícola, v. 34, p. 695-706, 2014.

MATIAS, S. S. R.; MARQUES JUNIOR, J.; PEREIRA, G. T.; SIQUEIRA, D. S. Ferramentas matemáticas, suscetibilidade magnética e modelos de paisagem aplicados na delimitação de áreas de manejo específico. Revista Brasileira de Ciência do Solo, v. 39, p. 968-980, 2015.

McKEAGUE, J. A.; DAY, J. H. Dithionite and oxalate-extractable Fe and Al as aids in differentiating various classes of soils. Soil Science Society of America Journal, v. 46, p. 13-22, 1966. 
MEHRA, O. P.; JACKSON, M. L. Iron oxide removal from soils by a dithionite-citrate system buffered with sodium bicarbonate. Clays and Clay Minerals, v. 7, p. 317-327, 1960.

MICHEL, F.M.; BARRÓN, V.; TORRENT, J.; MORALES, M.P.; SERNA, C.J.; BOILY, J.F.; LIU, Q.S.; AMBROSINI, A.; CISMASU, A.C.; BROWN, G.E. Ordered ferrimagnetic form of ferrihydrite reveals links among structure, composition, and magnetism. Proceedings of the National Academy of Sciences of the United States of America, v.107, n.7, 2787-2792, 2010.

OLIVEIRA, I.A.; MARQUES JUNIOR, J.; CAMPOS, M. C. C.; AQUINO, R. E.; FREITAS, L.; SIQUEIRA, D. S.; CUNHA, J. M. Variabilidade espacial e densidade amostral da suscetibilidade magnética e dos atributos de argissolos da região de Manicoré, AM. R. Revista Brasileira de Ciência do Solo, v. 39, p. 668-681, 2015.

PINGGUO, Y.; BYRNE, J. M.; YANG, M. Spatial variability of soil magnetic susceptibility, organic carbon and total nitrogen from farmland in northern China. Catena, v. 145, p. 92-98, 2016.

POGGERE, G. C.; INDA, A. V.; BARRÓ, V.; KÄMPF, N.; BRITO, A. D. B. de; BARBOSA, J. Z.; CURI, N. Maghemite quantification and magnetic signature of Brazilian soils with contrasting parent materials. Applied Clay Science, v. 161, p. 385-394, 2018.

RAMOS, P. V.; DALMOLIN, R. S. D.; MARQUES JUNIOR, J.; SIQUEIRA, D. S.; ALMEIDA, J. A. de; MOURA-BUENO, J. M. Magnetic Susceptibility of Soil to Differentiate Soil Environments in Southern Brazil. Revista Brasileira de Ciência do Solo, v. 41, p. e0160189, 2017.

ROSCOE, R.; BUURMAN, P.; VELTHORST, E. J. Disruption of soil aggregates by varied amounts of ultrasonic energy in fractionation of organic matter of a clay latosol: carbon, nitrogen, and $13 \mathrm{C}$ distribution in particle-size fractions. European Journal Soil Science, v. 51, p. 445-454, 2000.

SANTOS, H. L.; MARQUES JUNIOR, J.; MATIAS, S. R. R.; SIQUEIRA, D. S.; MARTINS FILHO M. V. Erosion factors and magnetic susceptibility in different compartments of a slope in Gilbués- PI, Brazil. Engenharia Agrícola, v. 33, p. 64-74, 2013.

SANTOS, R. D. dos; SANTOS, H. G. dos; KER, J. C.; ANJOS, L. H. C. dos; SHIMIZU, S. H. Manual de descrição e coleta de solo no campo. 7 ed. Sociedade Brasileira de Ciência do Solo: Viçosa, 2015.

SANTOS, H. G. dos; JACOMINE, P. K. T.; ANJOS, L. H. C. dos; OLIVEIRA, V. A. de; LUMBRERAS, J. F.; COELHO, M. R. et al. Sistema Brasileiro de Classificação de Solos. 5 ed. rev. e ampl. Brasília: Embrapa, 2018.

SILVA, J. E.; LEMAINSKI, J.; RESCK, D. V. S. Perdas de matéria orgânica e suas relações com a capacidade de troca catiônica em solos da região de cerrados do oeste baiano. Revista Brasileira de Ciência do Solo, v. 18. p. 541-547, 1994.

SILVA, A. R.; SOUZA JUNIOR, I. G.; COSTA, A. C. S. Suscetibilidade magnética do horizonte B de solos do Estado do Paraná. Revista Brasileira de Ciência do Solo, v. 34, n. 2, p. 329-338, 2010. 
SIQUEIRA, D. S.; MARQUES JUNIOR, J.; MATIAS, S. S. R.; BARRÓN, V.; TORRENT, J.; BAFFA, O.; OLIVEIRA, C. L. Correlation of properties of Brazilian Haplustalfs with magnetic susceptibility measurements. Soil Use and Management, v. 26, p. 425-431, 2010.

SIQUEIRA, D. S.; MARQUES JUNIOR, J.; PEREIRA, G. T.; TEIXEIRA, D. B.; VASCONCELOS, V.; CARVALHO JÚNIOR, A. O.; MARTINS, E.S. Detailed mapping unit design based on soil-landscape relation and spatial variability of magnetic susceptibility and soil color. Catena, v.135, p. 149$162,2015$.

SORENSEN, M. V.; STRIMBECK, R.; NYSTUEM, K. O.; KAPAS, R. E.; ENQUIST, B.; GRAAE, B. J. Draining the Pool? Carbon Storage and Fluxes in Three Alpine Plant Communities. Ecosystems, v. 21, n. 2, p. 316-330, 2018.

SOUZA, C. K.; MARQUES JUNIOR, J.; MARTINS FILHO, M. V.; PEREIRA, G. T.; Influência do relevo na variação anisotrópica dos atributos químicos e granulométricos de latossolo. Engenharia Agrícola, v.23, p. 486-495, 2003.

SOUZA JUNIOR, I. G.; COSTA, A. C. S.; VILAR, C. C.; HOEPERS, A. Mineralogia e susceptibilidade magnética dos óxidos de ferro do horizonte $B$ de solos do Estado do Paraná. Ciência Rural, $v$. 40, p. 513-519, 2010.

STRECK, E. V.; KÄMPF, N.; DALMOLIN, R. S. D.; KLAMT, E.; NASCIMENTO, P. C.; SCHNEIDER, P.; FLORES, C. A. Solos do Rio Grande do Sul. 3 ed. Porto Alegre: Emater/RS-Ascar, 2018.

TEIXEIRA, P. C; DONAGEMMA, G. K.; FONTANA, A.; TEIXEIRA, W. G. Manual de métodos de análise de solos. $3^{a}$. ed. rev. e ampl. - Brasília, DF: Embrapa; 2017.

TORRENT, J.; LIU, Q. S.; BARRÓN, V. Magnetic minerals in Calcic Luvisols (Chromic) developed in a warm Mediterranean region of Spain: Origin and paleoenvironmental significance. Geoderma, v. 154, p. 465-472, 2010.

WILDNER, W.; RAMGRAB, G. E.; LOPES, R. C.; IGLESIAS, C. M. F. Mapa Geológico do Estado do Rio Grande do Sul - Escala 1:750.000. CPRM - Serviço Geológico do Brasil: Porto Alegre, 2008.

YAMAMOTO, J. K.; LANDIM, P. M. B. Geoestatística: conceitos e aplicações. 1 ed. São Paulo: Oficina dos Textos, 2013.

ZHANG, L.; LIU, Y.; LI, X.; HUANG, L.; YU, D.; SHI, X.; Chen, H.; Xing, S. Effects of soil map scales on simulating soil organic carbon changes of upland soils in Eastern China. Geoderma, v. 312, p. 159-169, 2018. 


\section{Contribuição de autoria}

\section{1 - Priscila Vogelei Ramos}

Doutora em Ciências do Solo

priscilav.ramos@yahoo.com.br - https://orcid.org/0000-0002-6232-3441

Contribuição: Escrita - Primeira Redação, Escrita - Revisão e Edição

\section{2 - Ricardo Simão Diniz Dalmolin}

Doutor em Ciências do Solo

dalmolin@ufsm.br - https://orcid.org/0000-0002-8834-9869

Contribuição: Supervisão

\section{3 - Jean Michel Moura-Bueno}

Pós-doutorando Ciência do Solo

bueno.jean1@gmail.com - https://orcid.org/0000-0002-7240-3728

Contribuição: Escrita - Primeira Redação, Escrita - Revisão e Edição

\section{4 - José Marques Junior}

Doutor em Agronomia

marques@fcav.unesp.br - https://orcid.org/0000-0001-9317-586X

Contribuição: Supervisão

\section{5 - Diego Silva Siqueira}

Pós-graduado em Agronomia

diego_silvasiqueira@yahoo.com.br - https://orcid.org/0000-0003-3339-1143

Contribuição: Conceituação

\section{6 - Daniel De Bortoli Teixeira}

Doutor em Agronomia danielteixeira@unimar.br - https://orcid.org/0000-0001-8172-7911

Contribuição: Metodologia

\section{Como citar este artigo}

RAMOS, P.V; DALMOLIN, R. S. D.; MOURA-BUENO, J. M.; MARQUES JUNIOR, J.; SIQUEIRA, D. S.; TEIXEIRA, D. B. Suscetibilidade magnética na caracterização da variabilidade espacial de atributos do solo em solos subtropicais. Ciência e Natura, Santa Maria, v. 43, e47, p. 1-20, 2021. DOI 10.5902/2179460X41394. Disponível em: https://doi.org/10.5902/2179460X41394. Acesso em: dia mês abreviado. ano. 